\title{
Atomic lattice structures in two-dimensional momentum space
}

\author{
M. Rauner, S. Kuppens, M. Schiffer, G. Birkl, K. Sengstock, and W. Ertmer \\ Institut für Quantenoptik der Universität Hannover, Welfengarten 1, D-30167, Hannover, Germany
}

(Received 4 February 1998)

\begin{abstract}
In sub-Doppler laser cooling, the application of an additional homogeneous magnetic field gives rise to velocity selective resonances and laser cooling to nonzero velocities. We present a systematic investigation of the resulting structures in two-dimensional momentum space in an atomic beam experiment. The rms width of the resonances is close to one recoil momentum. The analysis of these structures in terms of a resonance condition shows good quantitative agreement with experimental results. [S1050-2947(98)51507-7]
\end{abstract}

PACS number(s): $32.80 . P j, 42.50 . \mathrm{Vk}, 32.80 . \mathrm{Lg}$

Transverse laser cooling of atomic beams in polarization gradient fields has become a powerful method to increase the phase-space density of atomic ensembles. In addition, atomic beams are widely used as tools for studying the interaction of light and matter. For example, it has been possible to determine diffusion constants, friction coefficients, and the influence of additional magnetic fields on laser cooling with optical molasses [1].

In particular, a magnetic field can have a significant influence on the dynamics of atomic motion in optical molasses. Besides magneto-optical trapping it can cause such effects as magnetic-field-induced laser cooling [2,3], velocity-selective resonances (VSRs) [4-8], or even an inhibition of subDoppler cooling $[7,9]$.

Cooling of an atomic beam to nonzero transverse velocities by VSR was observed in one dimension (1D) when a strong homogeneous magnetic field was applied [4,5]. This effect is due to enhanced Raman coupling of Zeeman substates when the Doppler shift compensates their energy splitting. Velocity-selective resonances in a three-dimensional optical lattice are reported by Triché et al. [10].

In our experiment, we transversely cool a slow atomic beam with a two-dimensional molasses light field that consists of two pairs of counterpropagating laser beams. The resulting two-dimensional (2D) atomic momentum distribution is detected in the far field with subrecoil resolution. This allows us to investigate different cooling mechanisms and their dynamics in detail, including the influence of additional external fields, such as dipole potentials [11]. In this Rapid Communication we report on sub-Doppler laser cooling in the presence of a homogeneous magnetic field. A typical example of the resulting momentum distribution is shown in Fig. 1(a). The complex lattice structure in momentum space is due to velocity-selective resonances, as will be discussed below.

A cold and slow beam of metastable neon atoms is prepared in a tilted magneto-optical funnel with additional subDoppler cooling $[12,13]$. The atomic beam has a high brilliance of $5 \times 10^{12}$ atoms/( $\left.\mathrm{sr} \mathrm{s}\right)$. The longitudinal velocity is $28 \mathrm{~m} / \mathrm{s}$ with an rms spread of $4 \mathrm{~m} / \mathrm{s}$. At the exit of the funnel, the beam radius is $42 \mu \mathrm{m}$. For our experimental geometry this constitutes a point source of atoms. The width of the initial velocity distribution can be varied between $2.5 v_{\text {rec }}$ and $10 v_{\text {rec }}$ with the recoil velocity $v_{\text {rec }} \equiv \hbar k / m=3.1 \mathrm{~cm} / \mathrm{s}$ (widths are given in Gaussian $\sigma$ throughout this paper). A velocity width of $10 v_{\text {rec }}$ corresponds to the Doppler cooling limit.

The atomic beam interacts with the transverse subDoppler molasses $10 \mathrm{~cm}$ downstream from the exit of the funnel. The atomic beam direction is normal to the plane defined by the $k$ vectors of the molasses beams. Two molasses configurations are of particular interest: the $\pi_{x} \pi_{y}$ and the $\sigma^{+} \sigma^{-}$configuration (Fig. 2). In the $\pi_{x} \pi_{y}$ sub-Doppler molasses the laser beams are linearly polarized with all polarization vectors within the plane of incidence $(x-y)$. The light field consists of two orthogonal standing waves with mutually perpendicular linear polarization. For a relative phase of $\pi / 2$ between these two waves, the resulting interference pattern is the $2 \mathrm{D}$ equivalent of the lin $\perp$ lin configuration for $1 \mathrm{D}$ molasses [14]. The $\sigma^{+} \sigma^{-}$configuration consists of two orthogonal pairs of beams with opposite circular polarization.
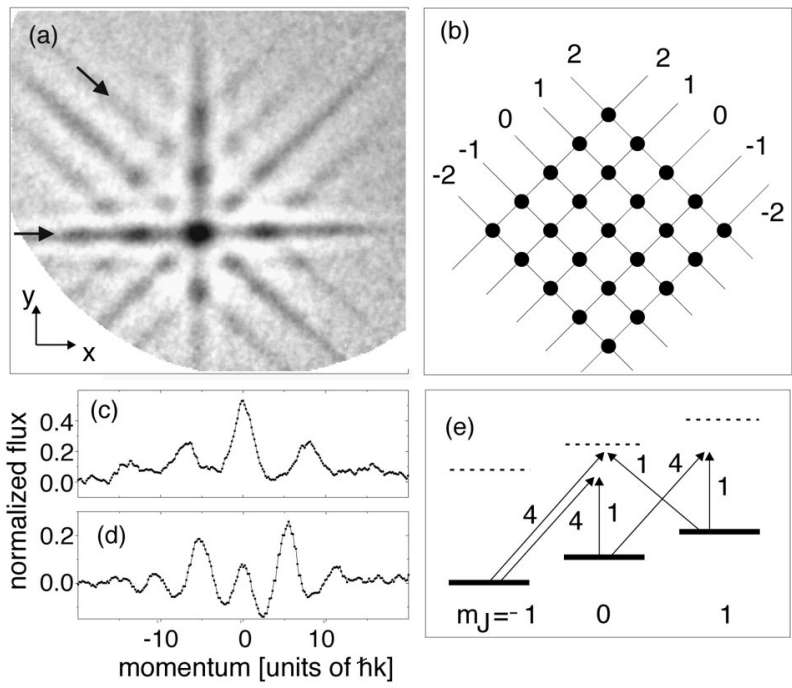

FIG. 1. Velocity-selective resonances in $2 \mathrm{D} \pi_{x} \pi_{y}$ molasses: B $=(-90,41,-180) \mathrm{mG}, \Delta=-9 \Gamma, s=2.6 \times 10^{-3}$. (a) Momentum distribution with dark regions representing increased atomic intensity. The full extension of the image is about $44 \hbar k$. (b) Expected VSR pattern. The horizontal and diagonal cross sections along the arrows in (a) are shown in (c) and (d), respectively. (e) Partial atomic energy diagram with two sets of resonances inducing Raman coupling for two VSR lines. The relative lengths of the arrows indicate different Doppler shifts (see text). 


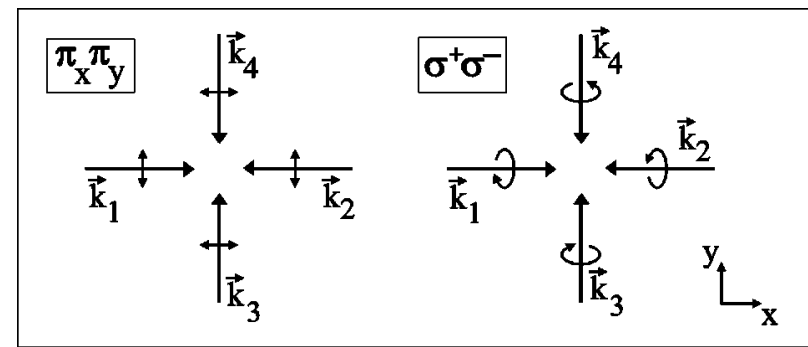

FIG. 2. Configuration of the 2D transverse cooling light field. The atomic beam is directed normal to the plane defined by the $k$ vectors. In the $\pi_{x} \pi_{y}$ configuration, all laser beams are linearly polarized in the plane of incidence. In the $\sigma^{+} \sigma^{-}$configuration, counterpropagating beams have opposite circular polarization.

The resulting light field has a spatial polarization structure that exhibits cooling features common to both $\operatorname{lin} \perp$ lin and $\sigma^{+} \sigma^{-}$cooling in $1 \mathrm{D}$.

The $3 s\left[\frac{3}{2}\right]_{2} \rightarrow 3 p\left[\frac{5}{2}\right]_{3}$ transition at $640 \mathrm{~nm}$ is used for laser cooling. The extent of the cooling region has been varied between 2 and $4 \mathrm{~mm}$. Knife edges define a nearly constantintensity light profile along the atomic beam direction in order to rule out possible adiabatic effects. With a mean longitudinal velocity of $28 \mathrm{~m} / \mathrm{s}$ this leads to interaction times between 70 and $140 \mu \mathrm{s}$. The light intensity is chosen so that the single beam saturation parameter $s=2 \Omega^{2} /\left(\Gamma^{2}+4 \Delta^{2}\right)$ is between $10^{-3}$ and $10^{-2}$, where $\Omega$ is the Rabi frequency, $\Gamma$ is the linewidth of the excited state, and $\Delta$ is the detuning from resonance. The total number of scattered photons is on the order of 100 per atom.

After a distance of $24 \mathrm{~cm}$ from the molasses, the metastable atoms are detected with high efficiency using a microchannel plate with an adjacent phosphor screen and chargecoupled-device camera. This directly gives a $2 \mathrm{D}$ image of the transverse atom distribution. The typical data acquisition time is several seconds to a minute. Since the time of flight of the atoms is well known, the observed spatial distribution is a map of the transverse momentum distribution. The implementation of velocity-selective detection results in a reduction of the longitudinal velocity spread to $v_{z} / \Delta v_{z}=40$. The overall transverse velocity resolution is about $0.6 v_{\text {rec }}$.

In order to gain information about the relative strength of the VSR we perform an image normalizing procedure. First, the image of the initial atom distribution is subtracted from the image of the cooled sample to give the net effect of the cooling process. The resulting image is then divided by the initial distribution for normalization. The normalized image thus gives the fractional increase or reduction of the atom intensity as a function of transverse momentum.

Figure 1(a) shows the transverse momentum distribution for the $\pi_{x} \pi_{y}$ configuration and a magnetic field of $206 \mathrm{mG}$ with components along $\hat{x}, \hat{y}$, and $\hat{z}$. The light regions represent a low number and the dark regions represent a high number of detected atoms. The uncooled momentum distribution is a Gaussian with a spread of $10 \hbar k$. The cooled distribution consists of two manifolds of diagonal lines of increased atom density and a point pattern of local maxima at the crossings of the lines.

For a quantitative analysis of the resonance velocities we follow the theoretical treatment of laser cooling in a strong magnetic field as outlined in Ref. [8] in terms of an operator formalism [17]. In a strong magnetic field, the eigenstates of the Hamiltonian are the Zeeman substates. The influence of the cooling light field is treated as a perturbation. The strongfield regime is defined by the condition $\gamma_{P} \ll \omega_{L}$, where $\gamma_{P}$ is the optical pumping rate and $\omega_{L}$ is the Larmor frequency. For typical molasses parameters, this requires a magneticfield strength that is larger than $100 \mathrm{mG}$.

When the atomic velocity is such that the sum of the Doppler shifts of two molasses beams is close to the energy splitting between two Zeeman ground states $|m\rangle$ and $\left|m^{\prime}\right\rangle$, the coupling of these states is strongly enhanced. As a consequence, atoms may also be cooled to nonzero velocities $\mathbf{v}$ by sub-Doppler cooling mechanisms. The general condition for these VSR is given by

$$
\left(\mathbf{k}_{i}-\mathbf{k}_{j}\right) \cdot \mathbf{v}-n \omega_{L}=0 .
$$

Here, $\mathbf{k}_{i, j}$ are the wave vectors of the molasses laser beams. The different resonances are labeled by $n \equiv m^{\prime}-m$. This equation describes a manifold of lines in $2 \mathrm{D}$ velocity space in which velocity-selective resonances occur. The range of values for $n$ depends on the angular momentum of the involved atomic levels and on the polarization components of the molasses beams with respect to the quantization axis set by the magnetic-field direction. For example, if, for an atom with ground-state angular momentum $J_{g} \geqslant 1$ and excitedstate angular momentum $J_{e}=J_{g}+1$, the orientation of the magnetic field is such that each molasses laser beam can induce $\pi, \sigma^{+}$, and $\sigma^{-}$transitions, then the maximum number of resonances with $n=0, \pm 1, \pm 2$ can occur. The case $n$ $=0$ is a Rayleigh resonance.

All of these resonances are visible in Fig. 1(a). Their position is consistent with Eq. (1) for a Larmor frequency $\omega_{L}$ $=2.4 \times 10^{6} \%$ s. Taking the experimental value of $|\mathbf{B}|=206$ $\mathrm{mG}$ we calculate $\omega_{L}^{\prime}=2.7 \times 10^{6} / \mathrm{s}$, in good agreement with the experiment. The $12 \%$ deviation is due to a residual magnetic-field gradient of about $40 \mathrm{mG} / \mathrm{cm}$ at the position of the interaction region and to uncertainties in the magneticfield measurement with a Gauss meter.

Sub-Doppler laser cooling is effective within the velocity capture range transverse to VSR lines. This causes the accumulation of atoms along these lines (dark regions) and the depletion of atom intensity for velocity classes in their vicinity (light regions). At the crossing of two VSR lines subDoppler cooling is effective in 2D and atoms are accumulated at VSR points. The global maximum in atom signal is at $v_{x}=0=v_{y}$.

The measured pattern can be understood from Fig. 1(b), where we have schematically depicted all diagonal resonances expected from Eq. (1). Since each laser beam can induce $\pi, \sigma^{+}$, and $\sigma^{-}$transitions for the magnetic-field direction chosen here, the complete set of resonances can be observed. For example, when an atom has a velocity, such that $k\left(v_{x}+v_{y}\right)=2 \omega_{L}$ with positive $v_{x}$ and $v_{y}$, then the $\sigma^{-}$ component of the red-shifted beam 1 (Fig. 2), together with the $\sigma^{+}$component of the blue-shifted beam 4 can couple Zeeman ground states with $\Delta m=2$. The same resonance is driven by the $\sigma^{-}$component of beam 3 and the $\sigma^{+}$component of beam 2. This gives rise to the VSR line $n=2$. Similarly, a $\pi$ polarized photon of beam 1 or 3 together with a $\sigma^{+}$ 

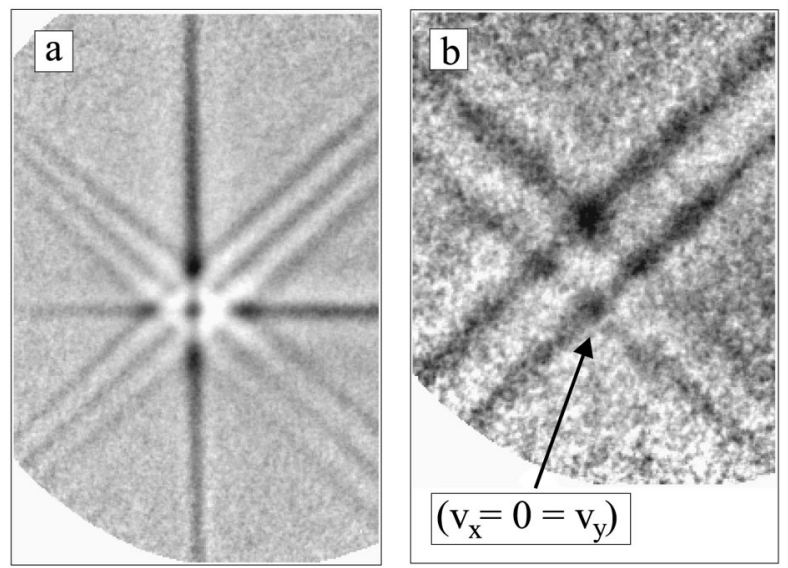

FIG. 3. VSR for (a) $\pi_{x} \pi_{y}$ with $\mathbf{B}=(0,100,0) \mathrm{mG}$ and (b) $\sigma^{+} \sigma^{-}$ with $\mathbf{B}=(-4,-181,-3) \mathrm{mG}$. In (a) the $\mathbf{v}=(0,0)$ point is the dark center point of the structure.

polarized photon of beam 4 , or a $\sigma^{-}$polarized photon of beam 2, respectively, can induce Raman coupling, which leads to the $n=1$ VSR line. Some of these processes are indicated in Fig. 1(e). Analogous situations give rise to the $n=-1$ and $n=-2$ VSR lines. The diagonal $n=0$ VSR lines are due to $\pi \pi$ transitions that transfer photons from one beam into an orthogonal one [8], both having the same Doppler shift.

Two cross sections of the normalized momentum distribution along the arrows indicated in Fig. 1(a) are shown in Figs. 1(c) and 1(d). The diagonal cross section [Fig. 1(d)] clearly shows five maxima in the momentum distribution. At the VSR points cooling enhances the peak atomic flux by up to $50 \%$ of the initial flux.

The momentum spread is similar for all VSR points and lines. It is determined by the optical pumping rate and the light shift in the same manner as for regular molasses cooling in the absence of a magnetic field [8]. Our experiments clearly confirm this prediction. Gaussian fits yield momentum widths between $1.2 \hbar k$ and $1.6 \hbar k$ (Gaussian $\sigma$ ). These values are equal to the momentum spread for the $\mathbf{v}=(0,0)$ peak in the absence of a magnetic field. Such surprisingly narrow structures with "temperatures" near the recoil limit have also been reported in $[3,13]$ in a similar context. We also observe lines with increased atom intensity along $\hat{x}$ and $\hat{y}$ with a width comparable to that of the diagonal VSR lines. These are not expected following the theory of polarization gradient cooling in a 2D $\pi_{x} \pi_{y}$ light field with a fixed relative phase of $\pi / 2[15,16]$. We attribute them to relative phases other than $\pi / 2$ present in our setup. This issue remains to be investigated in greater detail with phase stabilized molasses.

We observe no VSR lines for $2 k v_{i}= \pm \omega_{L}$ and $2 k v_{i}$ $= \pm 2 \omega_{L}(i=x, y)$. Although they are solutions of Eq. (1) there is no accumulation of atoms along these lines. The explanation for their absence is not obvious and involves a detailed investigation of the cooling dynamics: In the $\pi_{x} \pi_{y}$ configuration the cooling force that is given by the trace over the steady-state density matrix and the force operator is zero for $2 k v_{i}= \pm \omega_{L}$ and $2 k v_{i}= \pm 2 \omega_{L}$, because the steady-state density matrix has a constant population of the Zeeman levels for atoms moving along the $x$ or $y$ direction. There is no

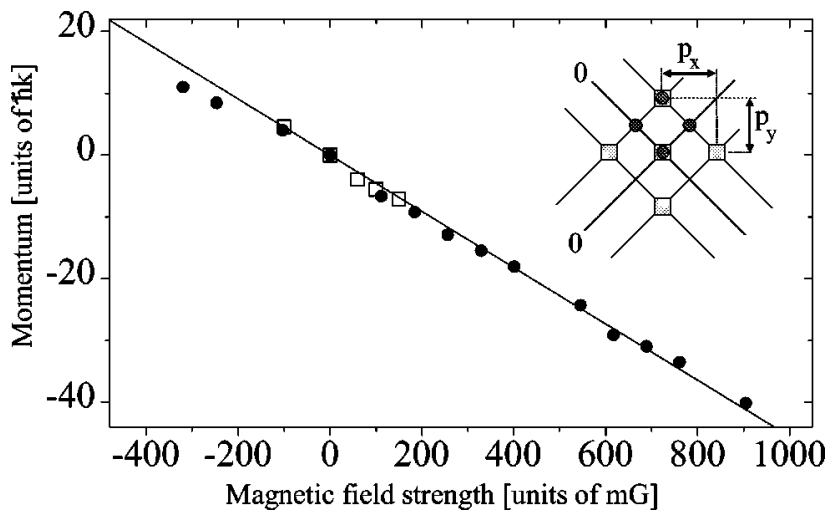

FIG. 4. Velocity-selective resonances as a function of the magnetic-field strength for the structures presented in Fig. 3. ( $\square$ ) momentum of the first VSR point along $\hat{x}$ for $\pi_{x} \pi_{y}$ molasses and $\Delta=-9 \Gamma$. (○) Momentum of the first VSR point along $\hat{y}$ for $\sigma^{+} \sigma^{-}$molasses and $\Delta=-3.5 \Gamma$. The solid line is the theoretical prediction. The inset indicates the plotted momentum components.

velocity-dependent population transfer between $m$ levels, which is essential for the cooling process.

Without a magnetic field, the momentum distribution consists of a single high-density spot at $v_{x}=0=v_{y}$ with four lines along $\hat{x}+\hat{y}, \hat{x}-\hat{y}, \hat{x}$, and $\hat{y}$. The diagonal lines have been predicted in the investigation of higher-dimensional molasses cooling $[15,16]$. They have been named escape channels, because laser cooling does not influence the velocity component along these lines.

We now turn to the discussion of the case in which $\mathbf{B}$ is directed along one of the laser beams. In this situation the structures strongly differ from that shown in Fig. 1(a). Depending on the polarization of the molasses beams and the orientation of the magnetic field, different subsets of the complete set of resonances are observed reflecting a reduction of the number of possible Raman couplings between Zeeman sublevels.

Figure 3(a) shows the momentum distribution for $\pi_{x} \pi_{y}$ molasses with a magnetic field of $100 \mathrm{mG}$ along $\hat{y}$. Only the diagonal VSR for $n=0, \pm 1$ and the horizontal and vertical lines through $v_{x}=0=v_{y}$ are visible. For this particular magnetic-field orientation, molasses beams 3 and 4 in Fig. 2 can each induce both $\sigma^{+}$and $\sigma^{-}$transitions, whereas beams 1 and 2 can only induce $\pi$ transitions. The diagonal VSR lines with $n= \pm 2$ vanish because they rely on $\sigma^{+} \sigma^{-}$or $\sigma^{-} \sigma^{+}$Raman coupling with one $\sigma$ photon from beam 1 or 2 . Reversing the magnetic field leads to an equivalent structure. This is expected, since for linearly polarized light the $\pi$ and $\sigma$ polarization projection onto the quantization axis is not altered by a change in the direction of the magnetic field.

In contrast, the VSR pattern for the $\sigma^{+} \sigma^{-}$configuration, with the $\mathbf{B}$ field along the direction of one of the laser beams, is not symmetric with respect to a reversal of $\mathbf{B}$. In Fig. 3(b) we show the momentum distribution of an atomic beam interacting with the $\sigma^{+} \sigma^{-}$molasses in a $181-\mathrm{mG}$ magnetic field mainly along $\hat{y}$. The diagonal VSR lines for $n=0$ and $n=+1$ are clearly visible. The polarization of beam 3 is purely $\sigma^{-}$, the polarization of beam 4 is purely $\sigma^{+}$, whereas beams 1 and 2 have all polarization components. For $k\left(v_{x}\right.$ $\left.+v_{y}\right)=\omega_{L}$ and positive $v_{x}$ and $v_{y}$, beams 2 and 3 and 
beams 1 and 4 can induce $\pi \sigma^{-}$and $\pi \sigma^{+}$Raman couplings, respectively. These cause the $n=+1$ resonance. The $n=$ +2 VSR lines are only vaguely visible. The $n<0$ resonances are absent, because there is no combination of molasses beams that could induce the necessary Raman coupling. For a $\mathbf{B}$ field of the same magnitude, but with a reversed direction the structure is reversed with respect to the $x$ axis. The observed resonances are then $n=0, n=-1$, and $n=$ -2 .

In order to verify the VSR condition [Eq. (1)] quantitatively, we have plotted in Fig. 4 the transverse momentum of the first VSR point along $\hat{x}$ of Fig. 3(a) and the transverse momentum of the first VSR point along $\hat{y}$ of Fig. 3(b) (see inset of Fig. 4) as a function of the $\mathbf{B}$ field amplitude. The resonance momentum scales linearly with the magnitude of B. The solid line shows the theoretical prediction of Eq. (1) based on an independent measurement of the magnetic field with a Gauss meter. The slope of the linear fit of the experi- mental data is in $10 \%$ agreement with the theoretical predictions.

The work presented in this paper demonstrates characteristic features of higher-dimensional laser cooling. Lattice structures in momentum space are generated by applying a magnetic field in addition to a 2D optical molasses. Depending on the magnetic-field orientation and the polarization state of the light, different subsets of the general resonance pattern can be observed. The application of this cooling scheme makes way for the generation of an atomic beam with multiple-peaked density distribution. The surprisingly narrow momentum widths, between $1.2 \hbar k$ and $1.6 \hbar k$, remain an interesting subject for future studies.

This work was supported by the Deutsche Forschungsgemeinschaft and the European Union (TMR Contract No. ERBFMRX-CT96-0002). S.K. acknowledges financial support from the Marie Curie Program of the EU.
[1] For an overview, see e.g., H. Metcalf and P. van der Straten, Phys. Rep. 244, 203 (1994).

[2] B. Sheehy, S.-Q. Shang, P. van der Straten, S. Hatamian, and H. Metcalf, Phys. Rev. Lett. 64, 858 (1990).

[3] M. D. Hoogerland, H. C. W. Beijerinck, K. A. H. van Leeuwen, P. van der Straten, and H. J. Metcalf, Europhys. Lett. 19, 669 (1992).

[4] S.-Q. Shang, B. Sheehy, H. Metcalf, P. v. d. Straten, and G. Nienhuis, Phys. Rev. Lett. 67, 1094 (1991).

[5] C. Valentin, M.-C. Gagné, J. Yu, and P. Pillet, Europhys. Lett. 17, 133 (1992).

[6] M. Walhout, J. Dalibard, S. L. Rolston, and W. D. Phillips, J. Opt. Soc. Am. B 9, 1997 (1992).

[7] J. Werner, H. Wallis, and W. Ertmer, Opt. Commun. 94, 525 (1992).

[8] P. van der Straten, S.-Q. Shang, B. Sheehy, H. Metcalf, and G. Nienhuis, Phys. Rev. A 47, 4160 (1993).
[9] M. Walhout, U. Sterr, and S. L. Rolston, Phys. Rev. A 54, 2275 (1996).

[10] C. Triché, F. Chevy, and G. Grynberg, preceding paper, Phys. Rev. A 58, 38 (1998).

[11] M. Schiffer, M. Rauner, S. Kuppens, M. Zinner, K. Sengstock, and W. Ertmer (unpublished).

[12] A. Scholz, M. Christ, D. Doll, J. Ludwig, and W. Ertmer, Opt. Commun. 111, 155 (1994).

[13] M. Schiffer, M. Christ, G. Wokurka, and W. Ertmer, Opt. Commun. 134, 423 (1997).

[14] A. Hemmerich and T. W. Hänsch, Phys. Rev. Lett. 70, 410 (1993).

[15] S. Marksteiner, K. Ellinger, and P. Zoller, Phys. Rev. Lett. 53, 3409 (1996).

[16] Y. Castin, K. Berg-Sørensen, J. Dalibard, and K. Mølmer, Phys. Rev. A 50, 5092 (1994).

[17] G. Nienhuis, P. van der Straten, and S.-Q. Shang, Phys. Rev. A 44, 462 (1991). 